\title{
Marked for ESRD
}

\section{By Lauren Martz, Staff Writer}

Researchers at the University of Miami Miller School of Medicine have found a biomarker-fibroblast growth factor 23-that could help predict whether a patient with early stages of kidney disease is at risk of progressing to and dying from end-stage renal disease. ${ }^{1}$ The team thinks the ability to pick out such patients could allow earlier intervention and could improve prognosis, but other researchers are not yet convinced the new biomarker offers such benefits.

Kidney disease is assessed by two primary measurements: estimated glomerular filtration ratio (GFR), which directly measures the ability of the kidney to filter the blood, and proteinuria, which measures the accumulation of proteins such as albumin in the urine as a result of kidney damage.

The measurements can help detect levels of kidney function and can assess the severity of damage or disease, but they are not necessarily able to indicate whether a patient's disease is likely to progress.

Previous studies identified fibroblast growth factor 23 (FGF23) as another indicator of kidney damage and showed that high levels of the hormone, which regulates phosphate levels, indicate increased risk of mortality in patients with end-stage renal disease (ESRD)., ${ }^{2,3}$ But it is difficult to alter the course of advanced disease,

"There is a demand for biomarkers for predialysis CKD patients. It could be helpful to know who is going to end up in dialysis and at end-stage renal disease. These patients could be treated earlier to prevent that progression."

-Myles Wolf,

University of Miami Miller School of Medicine and as a result, information on FGF23 levels offers only limited benefit to ESRD patients.

Now, Myles Wolf and colleagues at the University of Miami have found that high FGF23 levels can help determine whether patients in the early stages of chronic kidney disease (CKD) are likely to progress - a finding that could lead to better therapeutic outcomes.

Wolf is director of the Clinical Research Center and assistant dean for translational and clinical research at the University of Miami Miller School of Medicine.

The team also included researchers from The Children's Hospital of Philadelphia, the Perelman School of Medicine at the University of Pennsylvania, The Johns Hopkins University School of Medicine, The University of Alabama at Birmingham, St. John's Health System, the Tulane University School of Public Health and Tropical Medicine, Kaiser Permanente, the University of Michigan Health System, the Wayne State University School of Medicine, the University of California, San Francisco, the University of Illinois at Chicago and the NIH.
The researchers followed a group of 3,879 patients with stage 2-4 CKD for a median of 3.5 years. Patients in the highest quartile for FGF23 levels had a 4.3-fold higher risk of mortality than patients in the lowest quartile independent of other renal and cardiovascular factors.

In patients with mild to moderate CKD, FGF23 levels were significantly associated with increased risk of progression to ESRD. However, in patients who had already progressed to more severe stages of CKD, the strongest predictor for progression to ESRD was the GFR level itself. n these patients, FGF23 levels did not offer added predictive value.

The findings suggest FGF23 is best suited as a marker to predict both progression to ESRD and mortality in patients still in the earlier stages the disease who could better benefit from treatments.

Data were published in The Journal of the American Medical

\section{Biomarker validation}

Researchers were split on whether early knowledge of a patient's likelihood of progressing to ESRD will significantly change treatment decisions.

Currently, strategies for preventing or delaying further kidney damage in CKD patients involve controlling blood pressure and blood sugar. Patients with ESRD can also benefit from phosphate binders, which decrease the toxic levels of phosphorus that can accumulate in the blood from insufficient kidney function.

Thus, Paul Audhya, VP of development and CMO of Reata Pharmaceuticals Inc., told SciBX there are only limited measures that can be taken to alter the course of kidney disease at this time, which is why new biomarkers to predict risk have not been seen as particularly valuable.

Wolf, however, argued that "there is a demand for biomarkers for predialysis CKD patients. It could be helpful to know who is going to end up in dialysis and at end-stage renal disease. These patients could be treated earlier to prevent that progression."

For example, Wolf said the biomarker could be used to get CKD patients on a phosphate binder early in the course of disease. "Patients who already require dialysis have obvious reasons to go on these drugs. The benefit would really be to patients who might not be obvious candidates to benefit from phosphate binders but who may actually need them. Patients with high FGF23 but normal serum phosphate levels are still at risk for adverse effects, so it might be beneficial to start treating them with binders even though their serum phosphate levels are normal."

Audhya said it will be important to understand the added value of FGF23, or any other biomarker, beyond the risk for CKD progression already represented by the readily available biomarkers.

Reata is developing bardoxolone, an antioxidant inflammation modulator that induces a transcription factor called nuclear factor (erythroidderived 2)-like 2 (NFE2L2; NRF2). The compound is in Phase III testing to treat CKD. Audhya told SciBX that the company relied on estimated 
GFR to assess therapeutic effect in their Phase II study and will collect data for multiple biomarkers in order to better understand their role with regard to CKD progression and mortality in the Phase III trial.

In addition to validating the association in additional cohorts of patients and determining whether FGF23 measurements could provide additional information for treatment decisions for early stage patients, Richard Gregg, CSO of Vitae Pharmaceuticals Inc., suggested that the researchers might want to propose a cutoff point for increased ESRD and mortality risk. He said that cutoff could be helpful in the future for using FGF23 to assess a patient's prognosis.

Vitae's VTP-27999, a selective renin inhibitor, has completed Phase I testing to treat $\mathrm{CKD}$.

\section{Therapeutic target}

Another key step will be determining whether FGF23 directly affects the progression of $\mathrm{CKD}$, which could point to the hormone as a therapeutic target.

"It is also important to know whether this new target is just a biomarker or actually a mechanism of the disease plus a biomarker," said Wolf. "Maybe FGF23 is directly harmful itself, or maybe it is a biomarker for some other toxicity that is adversely affecting patients."

"The evidence to treat FGF23 levels by lowering the value does not yet exist," explained Audhya. "In fact, data on what to do with FGF23 are conflicting, as it was previously shown that vitamin D use, which is associated with increased FGF23 expression, was observed to be associated with improved clinical outcomes in kidney disease," he added.

Wolf said this is a complicated issue but declined to comment further.

"We see a general relationship between FGF23 and risk of death and kidney disease progression, but what I find most interesting is that when one controls for other factors, FGF23 is a stronger predictor for death than for progression of kidney disease," Gregg told SciBX. "We aren't yet clear whether this opens up new targets and pathways for intervention, whether it is directly related to kidney function or some disease processes and whether this can provide predictive value above and beyond that already provided by existing markers. This is one of those epidemiologic studies that doesn't necessarily answer many questions but certainly opens up a lot of very interesting new ones."

Wolf said the patent and licensing status of the work is not disclosed.

Martz, L. SciBX 4(28); doi:10.1038/scibx.2011.786

Published online July 21, 2011

REFERENCES

1. Isakova, T. et al. JAMA; published online June 15, 2011; doi:10.1001/jama.2011.826

Contact: Myles Wolf, University of Miami, Miami, Fla. e-mail:mwolf2@med.miami.edu

2. Gutiérrez, O.M. et al. N. Engl. J. Med. 359, 584-592 (2008)

3. Jean, G. et al. Nephrol. Dial. Transplant. 24, 2792-2796 (2009)

COMPANIES AND INSTITUTIONS MENTIONED

The Children's Hospital of Philadelphia, Philadelphia, $\mathrm{Pa}$. The Johns Hopkins University School of Medicine, Baltimore, Md. Kaiser Permanente, Oakland, Calif.

National Institutes of Health, Bethesda, Md.

Perelman School of Medicine at the University of Pennsylvania, Philadelphia, $\mathrm{Pa}$.

Reata Pharmaceuticals Inc., Irving, Texas

St. John's Health System, Detroit, Mich.

Tulane University School of Public Health and Tropical Medicine,

New Orleans, La.

The University of Alabama at Birmingham, Birmingham, Ala.

University of California, San Francisco, Calif.

University of Illinois at Chicago, Chicago, III.

University of Miami Miller School of Medicine, Miami, Fla.

University of Michigan Health System, Ann Arbor, Mich.

Vitae Pharmaceuticals Inc., Fort Washington, Pa.

Wayne State University School of Medicine, Detroit, Mich. 